\section{P60 IMMUNE STATUS TO MEASLES IN A U.S. LUPUS CLINIC SERVING PATIENTS WITH LIMITED ACCESS TO HEALTHCARE}

Alice Fike, Abhimanyu Amarnani, Yanira Ruiz-Perdomo, Sarfaraz Hasni, James Katz. NIAMS, Bethesda, New York, USA

\subsection{6/lupus-2020-eurolupus. 107}

Background Infections in patients with systemic lupus erythematosus (SLE) are a leading cause of morbidity and mortality. Preventive measures such as immunizations can reduce this burden. The United States experienced a surge in measles infections with outbreaks totaling more than 1,000 cases in 2019. Most of these outbreaks were associated with communities opposed to vaccination for varying reasons. Little attention has been given to communities at risk for measles due to limited access to health care. Current guidelines for screening prior to immune modulating therapy emphasize tuberculosis, opportunistic infections, viral hepatitis, and HIV, but do not discuss measles. Our clinic serves a majority Hispanic, Central American born population with limited access to healthcare. Measles seroprevalence studies of Central American countries have shown less than optimal rates of immunity. The CDC defines measles immunity as a positive titer, or evidence of 2 measles vaccines, or birth prior to 1957 . The population threshold for herd-immunity for measles is generally accepted to be $92-94 \%$ immune.

We seek to describe immune status to measles with current immunization status in our cohort of underserved patients with rheumatic diseases.

Methods Cross-sectional with a convenience sample of 95 patients with SLE born after 1957 who were seen in a community-health lupus clinic in 2019. All patients were participants in a natural history study of SLE. Titer for anti-rubeola IgG was requested for each patient with their routine clinical lab draw. Immunization records were requested from primary care providers for patients with negative or equivocal titers.

\begin{tabular}{|c|c|c|}
\hline & Immune* & Non-immune \\
\hline $\mathrm{N}$ & 84 & 11 \\
\hline Age, mean, SD & $44.9 \pm 9.1$ & $41.4 \pm 7.7$ \\
\hline \multicolumn{3}{|l|}{ Gender, $\%, n$} \\
\hline Male & $7(8.3 \%)$ & $1(9 \%)$ \\
\hline Female & $77(91.6 \%)$ & $10(90.9 \%)$ \\
\hline \multicolumn{3}{|l|}{ Ethnicity,\%, n } \\
\hline Hispanic or Latino & $51(60.7 \%)$ & $6(54.5 \%)$ \\
\hline Not Hispanic or Latino & $33(39.2 \%)$ & $5(45.4 \%)$ \\
\hline \multicolumn{3}{|l|}{ Race, $\%, n$} \\
\hline Black/African American & $16(19 \%)$ & $2(18.1 \%)$ \\
\hline White & $39(46.4 \%)$ & $2(18.1 \%)$ \\
\hline Asian & $9(10.7 \%)$ & $1(9 \%)$ \\
\hline Multiracial/Other & $20(23.8 \%)$ & $6(54.5 \%)$ \\
\hline \multicolumn{3}{|l|}{ Region of Birth, $\%, n$} \\
\hline USA & $19(22.6 \%)$ & $4(36.3 \%)$ \\
\hline Mexico and Central America & $35(41.6 \%)$ & $4(36.3 \%)$ \\
\hline South America and Caribbean & $18(21.4 \%)$ & $2(18.1 \%)$ \\
\hline Asia & $8(9.5 \%)$ & 0 \\
\hline Africa and Middle East & $4(4.7 \%)$ & $1(9 \%)$ \\
\hline
\end{tabular}

*Immune as defined as rubeola $\operatorname{lgG}>30 \mathrm{AU} / \mathrm{mL}$.
Information on demographics and willingness to be vaccinated in the future were collected.

Results We found evidence of sub-optimal levels of immunity within our cohort. Eleven patients $(11.5 \%)$ had negative or equivocal titers, and none had records documenting prior measles immunization. Only 2 of the 11 non-immune patients were eligible to receive a live vaccine and both of those patients indicated willingness to receive the MMR vaccine at a future visit. Given the small size of the non-immune group, our study was not sufficiently powered to detect differences across groups by region of birth.

Conclusions Our study shows sub-optimal levels of immunity to measles for our cohort of underserved patients with SLE and particularly highlighting missed opportunity for immunization prior to immunosuppression in patients with SLE. In this manner we expand the public health conversation concerning measles immunization in the United States. Not only is this an issue to address in anti-vaccination communities, but it is an important factor in communities with limited access to healthcare affected by rheumatic diseases such as SLE. Our results suggest that screening titers for measles should be considered by rheumatologists prior to the start of immunosuppression. Given that rheumatology patients living in communities with limited access to healthcare are at further risk due to their dysregulated immune systems and immunosuppressive therapies, both rheumatologists and primary care providers can reduce infection risk in these communities by updating immunizations in patients and family members.

\section{P61 DOES CELIAC DISEASE DIAGNOSIS MODIFY THE GAME RULES IN LUPUS PATIENTS? A 7 CASE SERIES REPORT}

Jorge Juan Fragío Gil, Roxana González Mazarío, José Ivorra Cortés, Elena Grau-García Luis González-Puig, Francisco Miguel Ortiz Sanjuan, Samuel Leal-Rodriguez, Isabel MartínezCordellat, Rosa Negueroles-Albuixech, José Eloy Oller-Rodríguez, Marta De-la-RubiaNavarro, Inmaculada Chalmeta-Verdejo, Cristina Alcañiz-Escandell, Cristóbal Pavez-Perales, Elvira Vicens-Bernabeu, Carmen Nájera-Herranz, Inés Cánovas-Olmos, José Andrés RománIvorra. Rheumatology Dept., La Fe Hospital, Valencia, Spain

\subsection{6/lupus-2020-eurolupus. 108}

Background/Purpose Autoimmune disorders tend to be aggregated since genetic, epigenetic and environmental pathogenic factors are usually common for several diseases. Specifically celiac disease (CD) is an uncommon comorbidity of Systemic Lupus Erythematosus (SLE), but seems to be extremely underdiagnosed.

We aimed to describe the SLE disease evolution after the CD diagnosis and the gluten free diet (GFD).

Methods An observational ambispective study including patients with prior diagnosis of SLE and posterior diagnosis of CD was performed. The moment since GFD was started was registered. Clinical characteristics of SLE were categorized like in RELESSER study. The first manifestation was considered only if counts in SLEDAI index.

Results 7 patients were included (all women) with a mean age of 45.14 (11.43) years old. SLE had a median evolution of $15(2.25,87)$ months. First manifestation of the disease was reported $48(24,84)$ months before. Domains mainly affected are mucocutaneous, hematological, articular and gastrointestinal. Six patients had history of recurrent aphtosis, which improved in all cases. Only 2 patients had positivity to anti- 
transglutaminase (IgA) antibodies. Six patients were DQ2 positive.

After being diagnosed of CD and starting the GFD, SLE patients seem to improve especially the leukopenia, lymphopenia and oral aphtosis, as well as SLEDAI score (showed in attached graphics).

Conclusions SLE patients with CD diagnosis and who started a GFD, showed improvement of leukopenia, lymphopenia, oral aphtosis and even SLEDAI.

In SLE patients with recurrent oral aphtosis and/or gastrointestinal unspecific symptoms, CD should be considered, but since serological screening displays a low sensitivity, HLA testing could be helpful. Gastroscopy should be considered, with biopsy and flow cytometer in uncertain cases. Even though, further studies, especially looking for different clinical profiles and longer observational period are needed.

\section{P62 POLY-AUTOIMMUNITY FREQUENCY IN SLE PATIENTS FROM A TERTIARY HOSPITAL}

Samuel Leal-Rodríguez, Elena Grau-García, Roxana González-Mazarío, Marta De-la-RubiaNavarro, Cristóbal Pávez-Perales, Isabel Martínez-Cordellat, Carmen Nájera-Herranz, Rosa Negueroles-Albuixech, José Eloy Oller-Rodríguez, Francisco Miguel Ortiz-Sanjuán, Elvira Vicens-Bernabeu, Cristina Alcañiz-Escandell, Inés Cánovas-Olmos, Inmaculada Chalmeta-Verdejo, Jorge Juan Fragio-Gil, Luis González-Puig, José Ivorra-Cortés, José Andrés Román-Ivorra. Rheumatology Dept., Hospital Universitario y Politécnico de La Fe, Valencia, Spain

\subsection{6/lupus-2020-eurolupus. 109}

Background/Purpose Poly-autoimmunity (PAI) is the presence of more than one Autoimmune Disease (AID) in one patient. The coexistence of Systemic Lupus Erythematosus (SLE) with other AIDs is a clinical challenge due to is one of the issues not yet elucidated in medical practice.

We aimed to determine PAI frequency in the context of SLE patients reported in a tertiary hospital.

Methods Cross-sectional observational study with systematic revision of electronic clinical records of SLE patients with other AIDs (from 2014 to 2018) was performed. Demographic, clinical and immunological data were collected.

Results Of 261 SLE patients, 48 (18.39\%) had PAI. Mean age was 51.19 (15.35) years $(93.75 \%$ were female). 2 patients from the $48(4.16 \%)$ had PAI with three AIDs. The $75 \%$ of cases developed SLE as the first AID. The mean age at diagnosis of the first AID was 35.52 (15.33) years and mean age at diagnosis of the second AID was 43.75 (16.31) years. A mean difference of 8.31 (9.24) years between the first and second AIDs debut was observed.

The most frequent AIDs registered that go along with SLE are Antiphospholipid Syndrome (APS)(39.58\%), Sjögren Syndrome (SS)(31.25\%), and Rheumatoid Arthritis (RA) (16.67\%). Moreover, in two cases a third AID was registered: SLE-SSAPS and SLE-APS-autoimmune-thyroiditis.

In the SLE-APS group, SLE was the AID of debut in the $89.47 \%$ of cases, instead of SLE-RA group with a $62.5 \%$. The SLE-APS group showed a $47.37 \%$ of cases with positive antiphospholipid antibodies and $64.71 \%$ positive lupus anticoagulant. In the SLE-RA group a $71.43 \%$ and $66.67 \%$ positive rheumatoid factor and antiCCP antibody was reported.

Conclusions $18.39 \%$ of patients with PAI in our group of SLE patients was observed, mostly with the SLE as the first AID developed. The most frequent association of AIDs in SLE cases were with APS, SS and RA.

\section{P63 ABNORMAL DISTRIBUTION OF CD27 ${ }^{+}$IGD $^{+}$ UNSWITCHED AND CD $27^{+}$IGD ${ }^{-}$SWITCHED MEMORY B CELLS IN SLE PATIENTS EXPOSED TO ORGANIC SOLVENTS}

${ }^{1}$ Carolina Hurtado, ${ }^{2}$ Diego Rojas-Gualdrón, ${ }^{2} E l s a$ María Vásquez-Trespalacios, ${ }^{3}$ Ricardo Pineda, ${ }^{4}$ Scott Jenks, ${ }^{5}$ Gloria Vásquez, ${ }^{4}$ Iñaki Sanz. ${ }^{1}$ School of Graduate Studies and School of Medicine, CES University, Medellin, Colombia; ${ }^{2}$ School of Medicine, CES University, Medellin, Colombia; ${ }^{3}$ Group of Clinical Information, Artmedica IPS, Medellin, Colombia; ${ }^{4}$ Dept. of Medicine, Division of Rheumatology, Lowance Center for Human Immunology, Emory University, Atlanta, USA; ${ }^{5}$ Grupo de inmunología celular e inmunogenética, Universidad de Antioquia, Medellin, Colombia

\subsection{6/lupus-2020-eurolupus.110}

Background Some studies in animal models, support an association between occupational exposure to Organic Solvents (OS) and Systemic Lupus Erythematosus (SLE). The specific physiopathological changes that these chemicals could induce to accelerate an autoimmune response are not known. Dysregulation of B cells is central in SLE, but very little is known on how OS exposure could influence it. This study aimed to examine the distribution of $\mathrm{B}$ cell subsets on Healthy Controls and SLE patients occupationally exposed to OS.

Methods 40 SLE patients who met ACR criteria and 17 Healthy Controls were recruited and classified as occupationally exposed or not to OS. Cryopreserved peripheral lymphocytes were analyzed by multiparametric Flow Cytometry using CD3, CD19, CD27, and IgD markers.

Results SLE patients exposed to OS had increased frequencies of CD27+ Switched Memory (SWM) cells. This change was associated with a specific OS like degreasers and ketones. Additionally, the few HC exposed to OS showed a decrease in Unswitched (USM) cells, with similar frequencies as those seen in SLE patients.

Conclusions Exposure to OS increased SWM cells on SLE patients and decreased USM cells on Healthy Controls. The influence of OS on SWM differentiation may be mediated through T cells. Previous reports of exposure to Trichloroethylene (a common OS), showed increased CD4+ T cell activation and secretion of INF- $\gamma$, this causes excessive $\mathrm{T}$ follicular helper development and germinal center formation in mice that could induce abnormalities in B cell subsets, and a similar mechanism may operate in OS exposed patients. Further research is needed to verify this hypothesis.

\section{P66 UTILIZATION OF GEOGRAPHIC INFORMATION SYSTEM (GIS) MAPPING TO ASSESS DISSEMINATION OF A LUPUS COMMUNITY BASED HEALTH AWARENESS MODEL}

${ }^{1}$ Karen Mancera-Cuevas, ${ }^{2}$ Daniel L Erickson, ${ }^{1}$ Anh Chung, ${ }^{2}$ Joan S Chmiel, ${ }^{3}$ Courtnie Phillip, ${ }^{3}$ Candace Feldman, ${ }^{4}$ Patricia Canessa, ${ }^{1}$ Rosalind Ramsey-Goldman. ${ }^{1}$ Medicine/ Rheumatology, Northwestern University, Chicago; ${ }^{2}$ Preventive Medicine, Northwestern University, Chicago; ${ }^{3}$ Division of Rheumatology, Brigham and Womans Hospital, Boston; ${ }^{4}$ Illinois Public Health Association, Springfield, USA

\subsection{6/lupus-2020-eurolupus.111}

Background We used a Popular Opinion Leader (POL) model, which leverages community leaders' social networks to disseminate health information and change norms in vulnerable communities. We established an academic-community partnerships in Chicago and Boston to increase knowledge about lupus and promote early care-seeking behaviors among African American 